\title{
Gastrodin Attenuates Tourette Syndrome by Regulating EAATs and NMDA Receptors in the Striatum of Rats
}

\section{Xueran Sun \\ Xin Zhang \\ Keyu Jiang \\ Min Wu}

Department of Traditional Chinese Medicine, Xinhua Hospital Affiliated to Shanghai Jiao Tong University School of Medicine, Shanghai, People's Republic of China
Correspondence: Min Wu Department of Traditional Chinese Medicine, Xinhua Hospital Affiliated to Shanghai Jiao Tong University School of Medicine, 1665 Kongjiang Road, Shanghai, 200092, People's Republic of China Tel + 86-1360I884805

Email wumin@xinhuamed.com.cn
Purpose: This study explored whether gastrodin (Gas) could attenuate the symptoms of Tourette syndrome(TS) via the regulation of glutamate (Glu), its transporters (EAAT1 and EAAT2) and its receptors (NMDAR1, NMDAR2A and NMDAR2B) in rats.

Materials and Methods: Seventy-five Wistar male rats were randomly divided into five groups ( $\mathrm{n}=15 \mathrm{each}$ ): the control, TS, Tia (tiapride, $25 \mathrm{mg} / \mathrm{kg}$ ), Gas60 (gastrodin, $60 \mathrm{mg} / \mathrm{kg}$ ) and Gas120 groups (gastrodin, $120 \mathrm{mg} / \mathrm{kg}$ ). Rats in all groups except the control group received intraperitoneal injection of 3,3'-iminodipropionitrile (IDPN) for 7 consecutive days to establish the TS model. Thereafter, rats in the Tia, Gas60, and Gas 120 groups were gavaged with $25 \mathrm{mg} / \mathrm{kg}$ Tia, $60 \mathrm{mg} / \mathrm{kg}$ Gas and $120 \mathrm{mg} / \mathrm{kg}$ Gas for 28 days. Rats in the control and TS groups were gavaged with $0.9 \%$ normal saline. Behavioral evaluation was performed by using stereotypy scoring, nodding experiment and autonomic activity test. The Glu level was measured by UPLC-QqQ-MS analysis. The expression of EAAT1, EAAT2, NMDAR1, NMDAR2A and NMDAR2B was measured by Western blot and quantitative real-time PCR (qRT-PCR) analyses.

Results: The results showed that rats with IDPN-induced TS exhibited an increase in stereotypy score, nodding numbers, number of times to enter the central area and autonomic total distance, which could be improved by Tia and Gas treatments. Furthermore, Tia and Gas treatments significantly decreased the IDPN-induced the increase in Glu levels in rats with TS. Furthermore, the decreased expression of EAAT1 and EAAT2 and increased expression of NMDAR1, NMDAR2A, and NMDAR2B in rats with TS induced by IDPN could be substantially altered by Tia and Gas treatments.

Conclusion: Gas ameliorated the behavioral dysfunction of rats with TS by maintaining Glu at a normal level, upregulating the expression of EAAT1 and EAAT2, and downregulating the expression of NMDAR1, NMDAR2A and NMDAR2B.

Keywords: Tourette syndrome, gastrodin, Glu, EAATs, NMDARs

\section{Introduction}

Tourette syndrome (TS), a chronic neuropsychiatric disorder with onset in childhood, is characterized by rapid, involuntary, repetitive, non-rhythmic, and burst-like phonic and motor tics. ${ }^{1,2}$ TS affects approximately $1 \%$ of school-age children, ${ }^{3-5}$ and boys are approximately three to four times more likely to develop the disease than girls. ${ }^{6}$ The majority of patients with TS may have other comorbidities, such as obsessive-compulsive disorder (OCD), attention-deficit/hyperactivity disorder (ADHD), autism, anxiety, and other emotional problems. ${ }^{7,8}$ Both tics and 
comorbidities may hinder the social interactions and learning of patients with TS and contribute to the overall level of functional impairment. ${ }^{9-11}$ Although the spectrum of treatment for TS has been expanding in recent years, the current treatment strategies are often unsatisfactory and have many adverse reactions. Therefore, it is necessary to seek novel treatments and drugs.

The etiology and pathogenesis of TS are still unclear. ${ }^{1}$ Previous studies have demonstrated that the etiology of TS is closely related to neurobiochemical factors, genetic factors, environmental factors, immunological factors, neuroinflammation, psychosocial factors, and other factors. ${ }^{12}$ Previous studies have found that abnormalities in the neurotransmission of glutamate (Glu) and gammaaminobutyric acid (GABA) are relevant to the pathogenesis of TS. ${ }^{13,14}$ Glu, the major neurotransmitter associated with TS, is the primary excitatory neurotransmitter of the central nervous system (CNS). ${ }^{15}$ It is widely accepted that there is a trend of an increase in Glu in cortico-striatothalamo-cortical (CSTC) circuits in patients with TS. ${ }^{16}$ Under physiological conditions, the excitatory amino acid transporters (EAATs) located in neurons and glial cells can take up Glu to maintain the concentration of extracellular Glu below excitotoxic levels. EAAT1 (glutamate aspartate transporter, GLAST) and EAAT2 (glial glutamate transporter, GLT-1) have a high affinity for Glu and may take up almost $80 \%$ of extracellular Glu, playing a key role in the uptake of Glu, termination of excitability signals and protection against neuronal damage. ${ }^{17}$ However, when EAATs dysfunction occurs or the release of Glu is excessive, more Glu gathers in the synaptic cleft, thus overactivating Glu receptors, which may lead to TS.

Extracellular Glu activates postsynaptic Glu receptors to regulate brain excitability. Among Glu receptors, NMDARs have been proved to be associated with the occurrence of TS. Three groups of NMDAR subunits exist: NMDAR1, NMDAR2 (further subdivided into four distinct members NMDAR2A- NMDAR2D) and more rarely NMDAR3 (further subdivided into two distinct members NMDAR3A and NMDAR3B). ${ }^{18,19}$ NMDAR1 plays a key role in the ion channel function of NMDAR, which is a main modulator of Glu. ${ }^{20}$ NMDAR2A and NMDAR2B are the main subunits expressed in forebrain regions (striatum, cortex, hippocampus). ${ }^{21}$ Previous studies have proved that GRIN2B, which encodes NMDAR2B, might be a susceptibility gene for neuropsychiatric disorders such as TS, OCD and ADHD. ${ }^{22}$ Therefore, we suggested that
NMDAR1, NMDAR2A and NMDAR2B might be closely related to the occurrence of TS.

Tian ma (also called Rhizoma Gastrodiae), the dried rhizome of Gastrodia elata Blume, is a notable traditional Chinese herb that has been used for the treatment of various conditions, including dizziness, headache, epilepsy, stroke, dementia, cardiovascular diseases, spasm, and other diseases for centuries. ${ }^{23}$ Gastrodin (Gas) is one of the major bioactive components isolated from the Rhizoma Gastrodiae. Numerous studies have shown that Gas possesses a broad range of pharmacological properties, including hypnotic, sedative, anti-tic, anti-vertigo, anxiolytic, anti-epileptic, antidepressant, and memoryimproving. ${ }^{24-27}$ It has been reported that Gas has beneficial effects on many CNS diseases, including TS, Parkinson's disease, Alzheimer's disease, epilepsy, affective disorders, cognitive impairment, and cerebral ischemia/reperfusion. ${ }^{23-25}$ The mechanisms of Gas include modulating neurotransmitters, anti-inflammatory, antioxidative, suppressing microglial activation, upregulating neurotrophins, and regulating mitochondrial cascades. $^{23,28,29}$ Although there have been some animal studies on the effect of Gas treating TS, ${ }^{24,25}$ few studies have demonstrated an association between the anti-tic effect of Gas and the functions of EAATs and NMDARs. This study was designed to explore the underlying mechanism of Gas in treating TS based on the EAATs and NMDARs functions. Collectively, we hypothesized that Gas might decrease the Glu level and regulate the expression of EAATs and NMDARs in rats with TS, thus playing a role in the treatment of TS.

In the present study, we evaluated the effects of Gas in a rat model of TS induced by 3,3'-iminodipropionitrile (IDPN) by behavioral tests, such as stereotypy scoring, nodding experiment, and autonomic activity test. The levels of Glu, GABA and dopamine (DA) were measured by UPLC-QqQ-MS. The protein and mRNA levels of EAAT1, EAAT2, NMDAR1, NMDAR2A, and NMDAR2B in the striatum of rats with TS were measured by Western blot and qRT-PCR.

\section{Materials and Methods Drugs and Reagents}

Gastrodin (Gas; Sigma-Aldrich, St. Louis, MO, USA), 3,3'Iminodipropionitrile (IDPN; Sigma-Aldrich, Shanghai, China), anti-EAAT1 (Abcam, Cambridge, MA, USA), antiEAAT2 (Abcam, Cambridge, MA, USA), anti-NMDAR1 
(Abcam, Cambridge, MA, USA), anti-NMDAR2B (Abcam, Cambridge, MA, USA), anti-NMDAR2A (ABclonal Technology, Wuhan, China), anti-GAPDH (ABclonal Technology, Wuhan, China), RIPA lysate (Beyotime, Nantong, China), and Bicinchoninic acid protein assay kit (BCA; Beyotime, Nantong, China) were used.

\section{Animals}

Seventy-five specific pathogen-free (SPF) Wistar male rats aged 3 weeks and weighing 50-60 g were purchased from Shanghai Sciple-Bikai Laboratory Animal Co., Ltd. (Shanghai, China, No: $\operatorname{SCXK}(\mathrm{Hu})$ 2017-0005). All rats were raised in an SPF animal room with a stable temperature of $22 \pm 2^{\circ} \mathrm{C}$, a relative humidity of $35 \pm 5 \%$, and a 12 hour light/night cycle environment. All rats had free access to food and water and were acclimatized for 7 days before experiments. All animal experiments were approved by the Laboratory Animal Ethical and Welfare Committee of Xinhua Hospital Affiliated to Shanghai Jiao Tong University of Medicine (XHEC-F-2021-04), which conforms to the guidelines from Directive 2010/63/EU of the European Parliament on the protection of animals used for scientific purposes. Animal pain was minimized during the experiment.

\section{Grouping and Administration of Animals}

All rats were randomly separated into 5 groups $(n=15$ in each group): control group, TS group, Tia group (tiapride, $25 \mathrm{mg} / \mathrm{kg}$ ), Gas60 group (gastrodin, $60 \mathrm{mg} / \mathrm{kg}$ ), Gas120 group (gastrodin, $120 \mathrm{mg} / \mathrm{kg}$ ). In the TS group, Gas60 group and Gas120 group, rats were injected intraperitoneally with $300 \mathrm{mg} / \mathrm{kg}$ IDPN once daily for 7 consecutive days to induce TS. Rats in control group were given an intraperitoneal injection with $0.9 \%$ normal saline. From the eighth day, rats in the Tia group, Gas60 group and Gas120 group were gavaged with $25 \mathrm{mg} / \mathrm{kg}$ Tia, $60 \mathrm{mg} / \mathrm{kg}$ Gas and $120 \mathrm{mg} / \mathrm{kg}$ Gas, respectively, once per day for subsequent 28 days. Rats in the control group and TS group were gavaged with an equal volume of $0.9 \%$ normal saline. An overview of the experimental procedures is shown in Figure 1.

\section{Behavioral Tests Stereotypy Scoring}

Stereotype scoring was performed by two trained independent examiners who were familiar with the experiments but blind to the allocation of groups. Under quiet and dark circumstances, the rats were reared in a separate observation cage. After 5 minutes of acclimation, the rats were scored by two observers for $5 \mathrm{~min}$ utes. The average score of each rat was calculated for statistical analysis. Stereotypy score was graded as follows: 0 points: no stereotyped movement; 1 point: rotation of body; 2 points: excessive up-and-down movement of the head and neck; 3 points: excessive upand-down movement and rotation of the head and neck; 4 points: head swings sideways and excessive up-anddown movement of the head and neck.

\section{Nodding Experiment}

Nodding experiment was performed by two trained independent examiners who were unaware of the allocation of groups. Under quiet and dark conditions, the rats were reared in a separate observation cage. After 5 minutes of acclimation, the nodding numbers of rats were counted and recorded by two observers for 5 minutes. The average nodding numbers of each rat were calculated for statistical analysis.

\section{Autonomic Activity Test}

The experiment was conducted in a quiet and shaded environment using an open field test box with dimensions of $100 \mathrm{~cm} \times 100 \mathrm{~cm} \times 50 \mathrm{~cm}$. The inner bottom and floor of the apparatus were black, and the bottom surface was equally divided into 25 squares. A camera connected with a computer was placed directly above the box, and the behavior of each rat was recorded for 5 minutes. The computer recorded the movement track and analyzed the number of times to enter the central area, central region residence time and total movement distance of each rat.

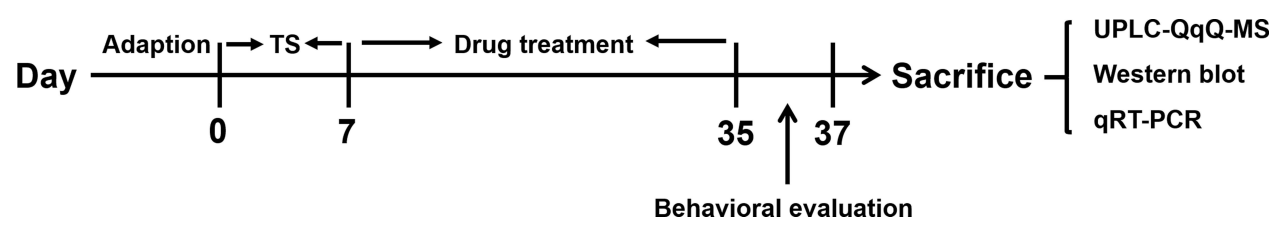

Figure I A schematic representation of the experimental procedures (TS model and treatments procedure in rats). 
The bottom and inner surfaces of the apparatus were cleaned with $75 \%$ ethanol after each test.

\section{Ultra-High Performance Liquid Chromatography Tandem Triple Quadruple Bar Mass Spectrometry (UPLC-QqQ-MS) Analysis}

UPLC-QqQ-MS was used to detect the levels of Glu, GABA and DA in the striatum of rats. In brief, the rat was deeply anaesthetized, and the whole striatum was dissected. The samples were stored in a freezer at $-80^{\circ} \mathrm{C}$. Before the measurement, the samples were taken from the freezer and thawed at room temperature. Then, $375 \mu \mathrm{L}$ of ice-cold sample extraction buffer (40\% methanol $+40 \%$ acetonitrile $+20 \%$ pure water) was added to each sample. After standing, the samples were lysed for $120 \mathrm{~s}$ to completely homogenize the tissue. The samples were centrifuged at $12,000 \mathrm{rpm}$ for $15 \mathrm{~min}$ at $4^{\circ} \mathrm{C}$, and the supernatants were transferred to new EP tubes. A total of $375 \mu \mathrm{L}$ of ice-cold sample extraction buffer was added to each sample residue. After shaking, the samples were centrifuged again. The supernatants were transferred to the previous supernatants. The supernatant was added with $300 \mu \mathrm{L}$ of chloroform, shaken, and placed in a freezer at $-20^{\circ} \mathrm{C}$. After $30 \mathrm{~min}$, the samples were centrifuged at $12,000 \mathrm{rpm}$ for $15 \mathrm{~min}$ at $4^{\circ} \mathrm{C}$. All supernatants were transferred to new EP tubes and diluted with sample diluent $(50 \%$ methanol $+50 \%$ pure water) to a proper concentration. Then, $50 \mu \mathrm{L}$ of the diluted sample solution was transferred to the injection vial for testing.

The analyses were performed by using ultraperformance liquid chromatography (H-Class UPLC) coupled with Xevo TQ-XS triple quadrupole MS (Waters, Milford, MA, USA). The separation was performed on an Acquity UPLC BEH Hilic column $(2.1 \mathrm{~mm} \times 100 \mathrm{~mm}, 1.7$ $\mu \mathrm{m})$ and the temperature of the column was wet at $40^{\circ} \mathrm{C}$. The mobile phase consisted of $0.25 \%$ formic acid aqueous containing $20 \mathrm{mmol}$ ammonium formate (solvent A) and $0.25 \%$ formic acid acetonitrile (93:7) containing $5 \mathrm{mmol}$ ammonium formate (solvent B). The injection volume was $1 \mu \mathrm{L}$. Under the selected electrospray ionization (ESI) conditions, analytes were detected by MRM in negative ionization mode. The capillary voltage was set at $3.5 \mathrm{kV}$. Nitrogen was used as the desolvation gas at a flow rate of $1000 \mathrm{~L} / \mathrm{h}$ at a temperature of $500^{\circ} \mathrm{C}$. The flow rate of the cone gas was set at $150 \mathrm{~L} / \mathrm{h}$.

\section{Western Blot Analysis}

Western blot was used to measure the protein levels of EAAT1, EAAT2, NMDAR1, NMDAR2A and NMDAR2B. Total proteins were extracted from the striatum of rats by using RIPA lysate, and the concentrations of the proteins were detected by using a BCA kit according to the manufacturer's instructions. The proteins were separated by $7.5 \%$ to $12.5 \%$ SDS-PAGE and then transferred onto polyvinylidene difluoride (PVDF) membranes (Millipore, Billerica, MA, USA). The PVDF membranes were blocked with 5\% skim milk for 2 hours at room temperature. Then, the membranes were incubated with the following appropriate concentrations of primary antibodies: anti-EAAT1 (1:1000), anti-EAAT2 (1:1000), anti-NMDAR1 (1:1000), anti-NMDAR2A (1:1000), antiNMDAR2B (1:1000) and anti-GAPDH $(1: 2000)$ at $4{ }^{\circ} \mathrm{C}$ overnight. The second day, the primary antibodies were discarded, the membranes were washed with TBST four times for $10 \mathrm{~min}$ each time. Then, the membranes were incubated with anti-mouse or anti-rabbit secondary antibodies at room temperature for 2 hours. After incubation with the secondary antibodies, the membranes were washed with TBST as described above. With enhanced chemiluminescence (ECL), the membranes were scanned by an imaging system (Bio-Rad, Hercules, CA, USA) to obtain protein bands. Then, the optical density of the protein band was quantified by using ImageJ software.

\section{Quantitative Real-Time Polymerase Chain Reaction (qRT-PCR) Analysis}

qRT-PCR was used to measure the mRNA levels of EAAT1, EAAT2, NMDAR1, NMDAR2A and NMDAR2B. Total RNA was extracted from the striatum of each rat by using TRIzol reagent (Invitrogen, Carlsbad, CA, USA) and reverse transcribed to synthesize cDNA by using a PrimeScript TM RT Master Mix kit (TaKaRa, Otsu, Japan) according to the manufacturers' instructions. Then, qRT-PCR was conducted by using a TB Green ${ }^{\circledR}$ Premix Ex Taq TM II kit (TaKaRa, Otsu, Japan). Thermal cycling conditions were as follow: $95^{\circ} \mathrm{C}$ for 30 s, followed by 40 cycles of $95^{\circ} \mathrm{C}$ for $5 \mathrm{~s}$ and $60^{\circ} \mathrm{C}$ for 30 s. The gene expression of GAPDH was used as an internal reference, and all gene expression values were normalized against the same samples. The results were quantitatively analyzed according to the $2^{-\Delta \Delta \mathrm{Ct}}$ method. All the sequences of primers are shown in Table 1 . 
Table I Primer Sequences Used for the qRT-PCR Analysis

\begin{tabular}{|c|c|c|}
\hline Gene & Sequences $\left(5^{\prime}-3^{\prime}\right)$ & \\
\hline EAATI & $\begin{array}{l}\text { Forward } \\
\text { Reverse }\end{array}$ & $\begin{array}{l}\text { GCCATCATGAGATTGGTAGCGGT } \\
\text { GGAAGTAGAGGAGAGGCAGGACGA }\end{array}$ \\
\hline ЕАAT2 & $\begin{array}{l}\text { Forward } \\
\text { Reverse }\end{array}$ & $\begin{array}{l}\text { GAACTTCGGTCAATGTAGTGGGCG } \\
\text { TGGACTGCGTCTTGGTCATTTCG }\end{array}$ \\
\hline NMDARI & $\begin{array}{l}\text { Forward } \\
\text { Reverse }\end{array}$ & $\begin{array}{l}\text { CGGTATCAGGAATGCGACTC } \\
\text { GGAAAATCCCAGCTACGATG }\end{array}$ \\
\hline NMDAR2A & $\begin{array}{l}\text { Forward } \\
\text { Reverse }\end{array}$ & $\begin{array}{l}\text { GCTTGTGGTGATCGTGCTGAA } \\
\text { AATGCTGAGGTGGTTGTCATCTG }\end{array}$ \\
\hline NMDAR2B & $\begin{array}{l}\text { Forward } \\
\text { Reverse }\end{array}$ & $\begin{array}{l}\text { TGGCTATCCTGCAGCTGTTTG } \\
\text { TGGCTGCTCATCACCTCATTC }\end{array}$ \\
\hline GAPDH & $\begin{array}{l}\text { Forward } \\
\text { Reverse }\end{array}$ & $\begin{array}{l}\text { TTCCTACCCCCAATGTATCCG } \\
\text { CСACССTGTTGCTGTAGCCATA }\end{array}$ \\
\hline
\end{tabular}

\section{Statistical Analysis}

All statistical analyses were performed using SPSS 20.0 software (IBM, Armonk, NY, USA). All the experimental data are normally presented as the mean \pm SD. One-way analysis of variance (ANOVA) was used to statistically analyze the experimental results, and the differences among groups were compared using multiple least significant difference (LSD) tests. $p<0.05$ was considered statistically significant. All figures were obtained by GraphPad Prism 8.0 software (GraphPad Software Inc., San Diego, CA, USA).

\section{Results}

\section{Effects of Gas on the Behaviors of Rats with TS}

For the stereotypy scoring, as shown in Figure 2, there was a significant increase in the stereotypy score of the rats in the TS group $(3.60 \pm 0.63, p=0.000)$ compared with the control group. However, treatment with Tia $(1.07 \pm 0.59, p=0.000)$, Gas60 (2.40 $\pm 0.51, p=0.000)$ and Gas120 (1.20 $\pm 0.56, p=0.000)$ substantially decreased the stereotypy score of the rats compared with that of the TS group.

For the nodding experiment, as shown in Figure 3, the nodding numbers of the rats in the TS group (84.80 \pm 5.39 , $p=0.000$ ) showed a significant increase compared with those of the control group $(20.60 \pm 3.72)$. However, treat-

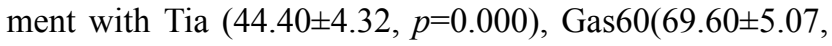
$p=0.000)$ and Gas $120(50.20 \pm 5.70, p=0.000)$ strongly decreased the nodding numbers of the rats compared with those of the TS group.
For the autonomic activity test, as shown in Figure 4A$\mathrm{D}$, the number of times the rats entered the central area (26.07 $\pm 11.20, p=0.000)$ and the autonomic total distance travelled (43041.51 $\pm 7831.83 \mathrm{~mm}, p=0.000)$ were significantly greater in the TS group than in the control group $(10.53 \pm 6.29 ; 19570.55 \pm 2376.58 \mathrm{~mm})$. However, the number of times the rats entered the central area and the autonomic total distance travelled were markedly decreased after treatment with Tia $(12.80 \pm 9.02, p=0.000$;

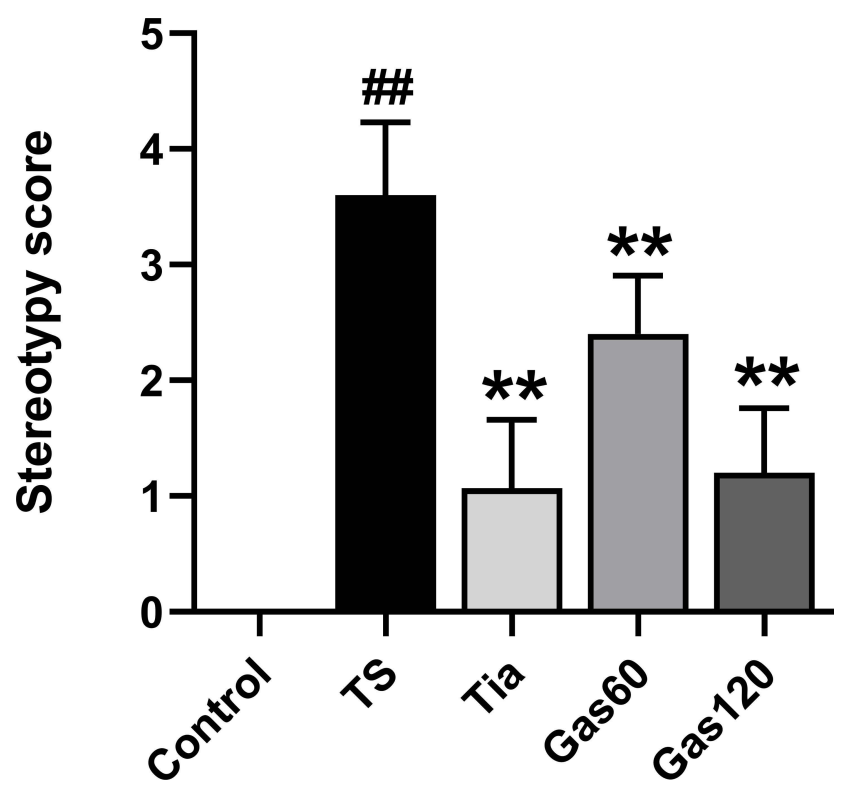

Figure 2 Effects of Gas treatment on stereotypy score of rats with TS induced by IDPN.

Notes: Stereotypy score was measured as described in materials and methods. Each bar represented the mean $\pm S D(n=15) .{ }^{\#} p<0.0$ I vs control group; $* *<<0.0$ I vs TS group. 


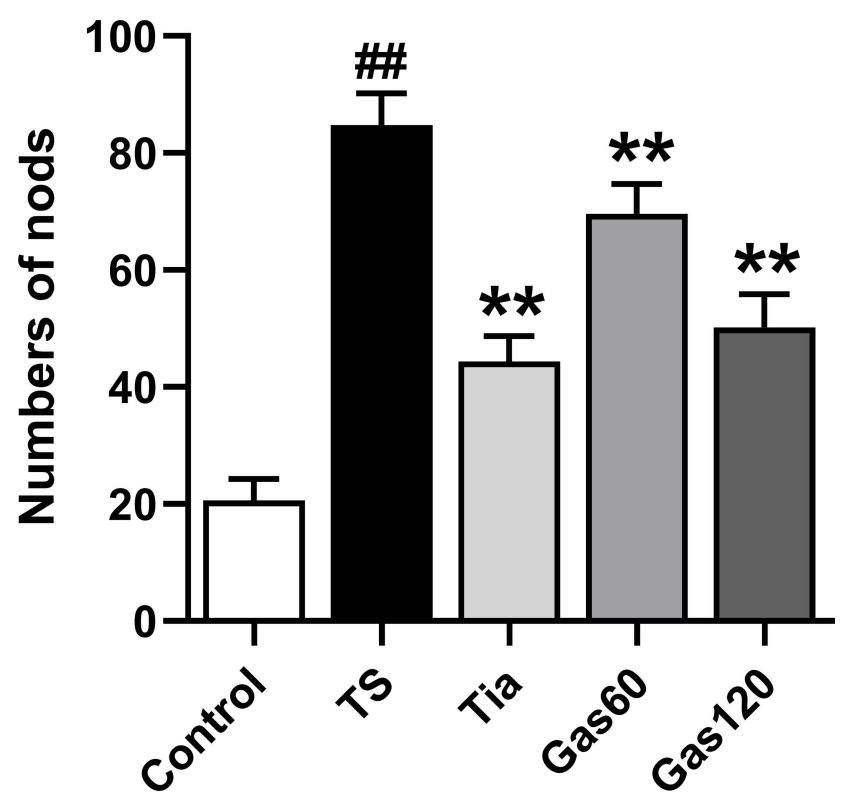

Figure 3 Effects of Gas treatment on nodding numbers of rats with TS induced by IDPN.

Notes: Numbers of nods were measured as described in materials and methods. Each bar represented the mean $\pm S D(n=15) .{ }^{\#} p<0.01$ vs control group; $* * p<0.01$ vs TS group.

$25567.68 \pm 2675.04 \mathrm{~mm}, p=0.000), \operatorname{Gas} 60 \quad(18.73 \pm 12.35$, $p=0.035 ; 34630.45 \pm 1802.88 \mathrm{~mm}, p=0.009)$ and Gas 120 (15.33 $\pm 6.17, p=0.002 ; 27815.35 \pm 2389.80 \mathrm{~mm}, p=0.000)$ compared with those of the TS group. The differences in the central region residence time of the rats in the control, TS, Tia, Gas60 and Gas120 groups were not significant $(p>0.05)$.

Effects of Gas on the Glu, GABA and DA Levels in the Striatum of the Rats with TS As shown in Figure $5 \mathrm{~A}$ and $\mathrm{B}$, the Glu levels in the TS group $(224.46 \pm 12.43 \mu \mathrm{g} / \mathrm{mL}, p=0.000)$ were significantly higher than those in the control group $(118.79 \pm 7.39 \mu \mathrm{g} /$ $\mathrm{mL})$. However, the Glu levels in the Tia group (154.09 $\pm 11.11 \mu \mathrm{g} / \mathrm{mL}, p=0.000)$, Gas60 group $(197.01 \pm 9.80 \mu \mathrm{g} /$ $\mathrm{mL}, p=0.002)$ and Gas120 group $(172.17 \pm 11.30 \mu \mathrm{g} / \mathrm{mL}$, $p=0.000$ ) were substantially decreased compared with those in the TS group.

As shown in Figure $\mathrm{S} 1 \mathrm{~A}$ and $\underline{\mathrm{B}}$, we found increased GABA and DA levels in the TS group (GABA: 45.50 $\pm 2.80 \mu \mathrm{g} / \mathrm{mL}, \quad p=0.000 ;$ DA: $2423.25 \pm 131.86 \mu \mathrm{ng} / \mathrm{mL}$, $p=0.000$ ) compared with the control group (GABA: $34.13 \pm 2.06 \mu \mathrm{g} / \mathrm{mL} ; \quad \mathrm{DA}: \quad 1778.75 \pm 90.64 \quad \mathrm{ng} / \mathrm{mL})$. However, the differences in the levels of GABA and DA of the rats in the TS, Gas60 and Gas 120 groups were not significant $(p>0.05)$.
Effects of Gas on the Protein Expression of EAATI, EAAT2, NMDARI, NMDAR2A, and NMDAR2B in the Striatum of the Rats with TS

As shown in Figure $6 \mathrm{~A}-\mathrm{C}$, the relative protein expression levels of EAAT1 and EAAT2 in the striatum of the rats in the TS group (EAAT1: $0.38 \pm 0.05, p=0.000$; EAAT2: 0.32 $\pm 0.03, p=0.000$ ) were obviously lower than those in the striatum of the rats in the control group (EAAT1: 1.00 \pm 0.04 ; EAAT2: $1.00 \pm 0.01$ ). However, the protein levels of EAAT1 and EAAT2 in the Tia (EAAT1: $0.65 \pm 0.03$, $p=0.000$; EAAT2: $0.83 \pm 0.02, p=0.000$ ), Gas60 (EAAT1: $0.53 \pm 0.01, p=0.000$; EAAT2: $0.45 \pm 0.03, p=0.000)$ and Gas120 (EAAT1: $0.86 \pm 0.02, p=0.000 ; \quad$ EAAT2: 0.67 $\pm 0.00, p=0.000)$ groups were significantly increased relative to those in the TS group.

As shown in Figure 6A, D-F, the relative protein expression levels of NMDAR1, NMDAR2A and NMDAR2B in the striatum of the rats in the TS group (NMDAR1: $2.24 \pm 0.12, p=0.000$; NMDAR2A: $2.33 \pm 0.03$, $p=0.000$; NMDAR2B: $4.08 \pm 0.10, p=0.001)$ were markedly higher than those in the control group (NMDAR1: 1.00 \pm 0.06 ; NMDAR2A: $1.00 \pm 0.02$; NMDAR2B: $1.00 \pm 0.03$ ). However, the upward trend was obviously weakened by treatment with Tia (NMDAR1: $1.38 \pm 0.10, p=0.000$; NMDAR2A: $1.21 \pm 0.02, \quad p=0.000 ; \quad$ NMDAR2B: 1.84 $\pm 0.02, p=0.002$ ), Gas60 (NMDAR1: $1.89 \pm 0.06, p=0.000$; NMDAR2A: $1.75 \pm 0.10, p=0.000$; NMDAR2B: $2.67 \pm 0.16$, $p=0.003$ ) and Gas120 (NMDAR1:1.65 $\pm 0.04, p=0.000$; NMDAR2A:1.34 $\pm 0.07, p=0.000 ;$ NMDAR2B:1.24 \pm 0.08 , $p=0.000)$ compared with that of the TS group.

\section{Effects of Gas on the mRNA Expression of EAATI, EAAT2, NMDARI, NMDAR2A, and NMDAR2B in the Striatum of the Rats with TS}

As shown in Figure 7A and B, there was a downward trend in the relative mRNA expression levels of EAAT1 and EAAT2 in the striatum of the rats in the TS group (EAAT1: $0.33 \pm 0.12, p=0.000$; EAAT2: $0.37 \pm 0.07, p=0.000)$ compared with the control group (EAAT1: $1.00 \pm 0.14$; EAAT2: 1.00 $\pm 0.15)$. However, the decreased mRNA levels of EAAT1 and EAAT2 were significantly altered in the Tia (EAAT1: 0.67 $\pm 0.05, \quad p=0.004$; EAAT2: $0.67 \pm 0.10, \quad p=0.002)$, Gas60 (EAAT1: $0.63 \pm 0.11, p=0.009$; EAAT2: $0.61 \pm 0.07, p=0.01$ ) 


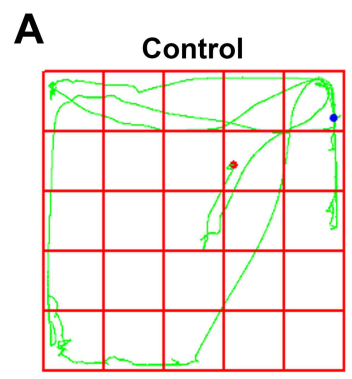

B

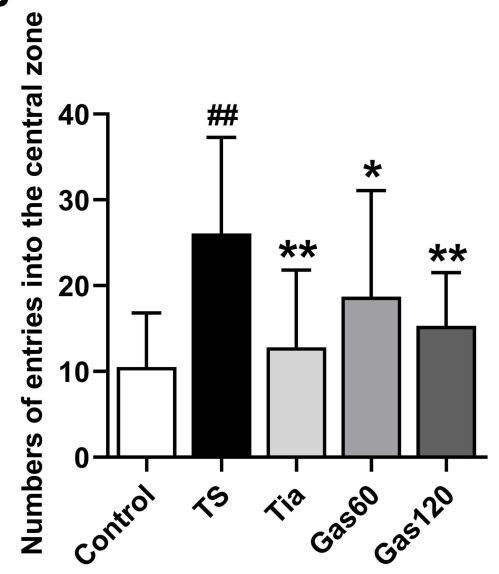

TS

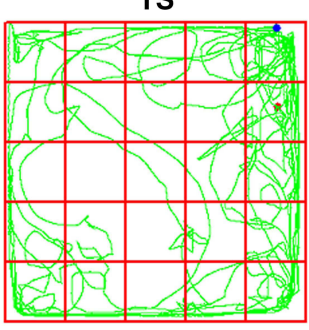

Tia

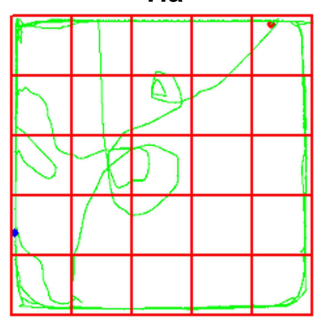

Gas60

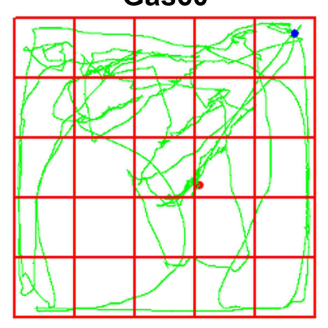

Gas120

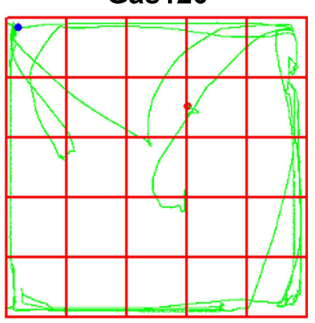

C

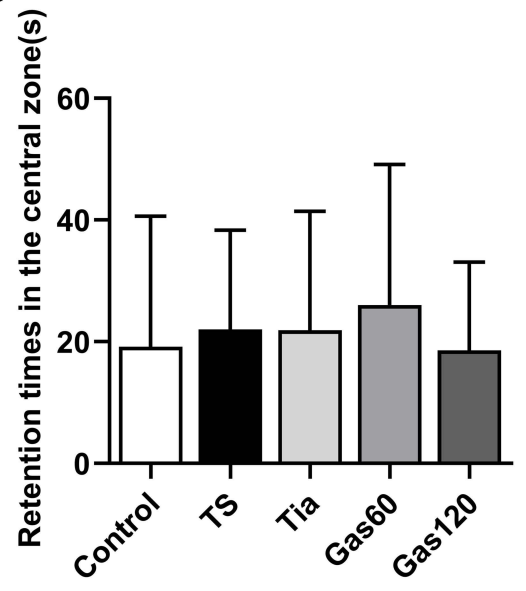

D

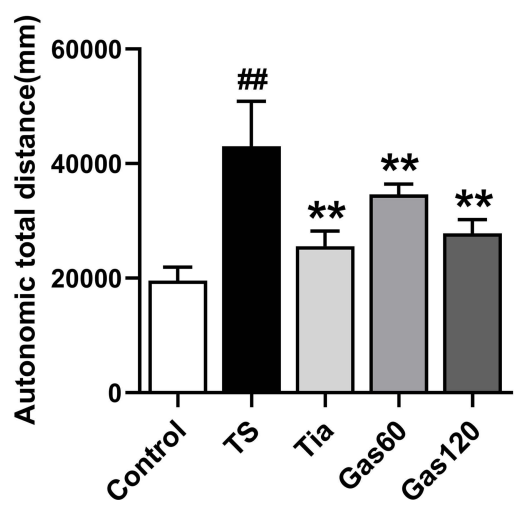

Figure 4 Effects of Gas treatment on autonomic activity of rats with TS induced by IDPN.

Notes: (A) Representative movement trajectory of each group of rats. (B) The number of times to enter the central area of each group of rats. (C) The central region residence time of each group of rats. (D) The autonomic total distance of each group of rats. The movement trajectory, number of times to enter the central area, central region residence time and autonomic total distance were detected as described in materials and methods. Each bar represented the mean \pm SD ( $n=I 5)$. ${ }^{\prime} p<0.0 \mathrm{I}$ vs control group; $* p<0.05, * * p<0.01$ vs TS group.

and Gas120 (EAAT1: $0.69 \pm 0.13, p=0.003 ;$ EAAT2: 0.76 $\pm 0.03, p=0.000)$ groups relative to the TS group.

As shown in Figure 7C-E, the relative mRNA expression levels of NMDAR1, NMDAR2A and NMDAR2B in the striatum of the rats in the TS group (NMDAR1: $1.62 \pm 0.13$, $p=0.000$; NMDAR2A: $1.74 \pm 0.09, p=0.000$; NMDAR2B: $1.89 \pm 0.11, p=0.000)$ presented an upward trend compared with those of the control group (NMDAR1: $1.00 \pm 0.15$; NMDAR2A: $\quad 1.00 \pm 0.07$; NMDAR2B: $\quad 1.00 \pm 0.21)$. Treatment with Tia (NMDAR1: $1.09 \pm 0.05, p=0.000$; NMDAR2A: $1.01 \pm 0.08, p=0.000$; NMDAR2B: $1.15 \pm 0.19$, $p=0.001$ ) and Gas120 (NMDAR1: $1.29 \pm 0.14, p=0.009$; NMDAR2A: $1.18 \pm 0.18, p=0.000$; NMDAR2B: $1.32 \pm 0.28$, $p=0.007)$ substantially reversed the TS-induced alternation in the mRNA expression of NMDAR1, NMDAR2A and NMDAR2B compared with that of the TS group. There was a significant decrease in NMDAR2A relative mRNA expression in the Gas60 group $(1.44 \pm 0.12, p=0.01)$ relative to the TS group. However, the differences between the TS group and the Gas60 group in NMDAR1 and NMDAR2B mRNA relative expression were not significant $(p>0.05)$.

\section{Discussion}

The purpose of this study was to investigate the anti-tic effect of Gas on rats with TS induced by IDPN and explore the potential mechanisms underlying these antitic effects. We found that Gas could alleviate the behavioral symptoms of rats with TS induced by IDPN including stereotypy score, nodding numbers, and autonomic activity. More importantly, the Gas mediated amelioration of the behavioral dysfunction of rats with TS might be associated with the maintenance of Glu at a normal level, upregulation of the expression of EAAT1 and EAAT2, and downregulation of the expression of NMDAR1, NMDAR2A and NMDAR2B.

The choice of animal model plays a crucial role in the study of new therapeutic strategies and the pathogenesis of diseases. IDPN is a neurotoxin that is commonly used to establish TS animal models. ${ }^{30}$ In this study, stereotypy scoring, nodding experiment, and autonomic activity test were conducted to access the severity of behavioral symptoms of rats with TS induced by IDPN. The stereotypy 

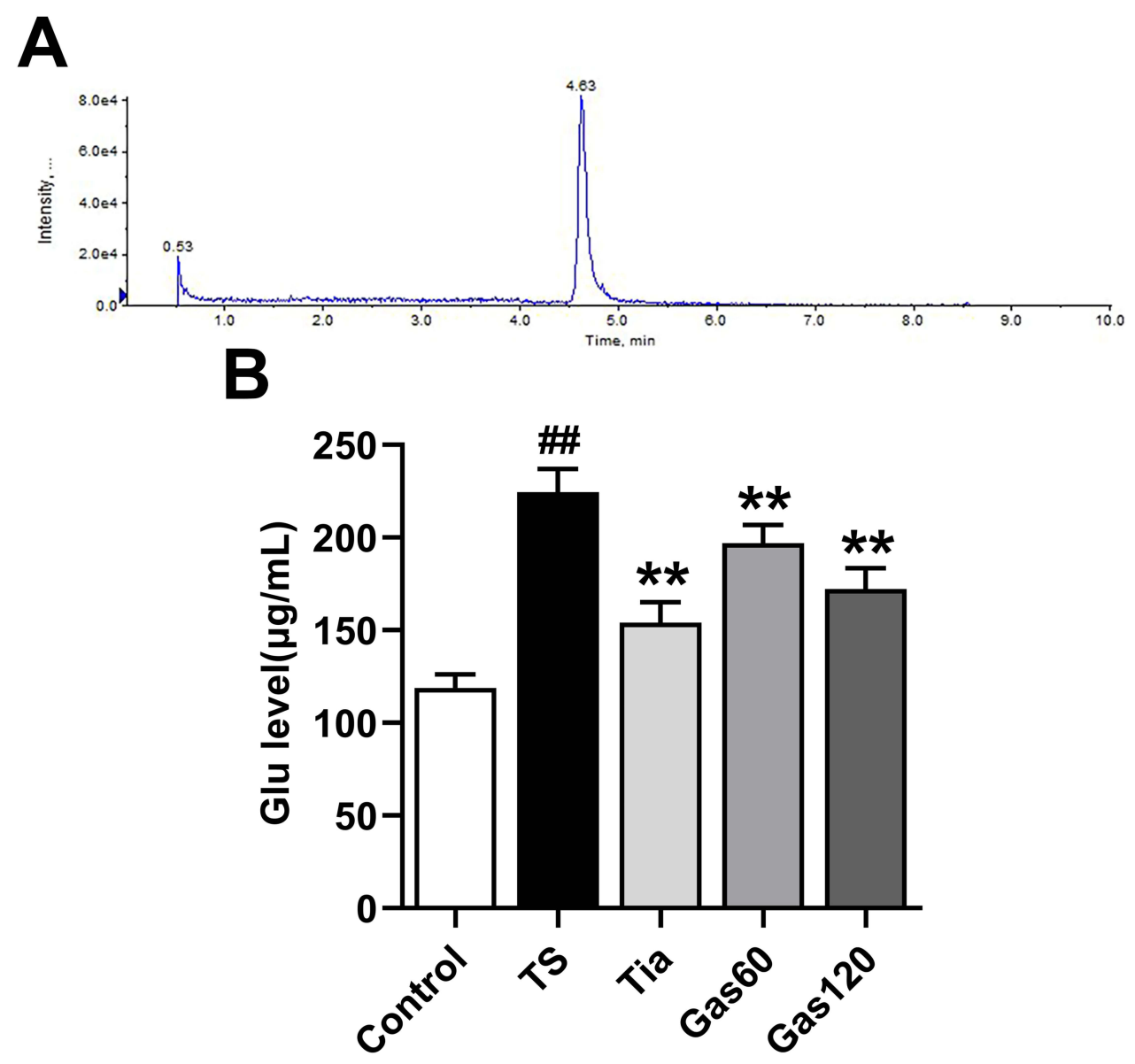

Figure 5 Effects of Gas treatment on Glu level in the striatum of the rats with TS induced by IDPN.

Notes: (A) Representative extracted chromatograms of Glu. (B) The Glu level of each group of rats. Glu level was measured as described in materials and methods. Each bar represented the mean $\pm S D(n=4) .{ }^{\#} p<0.0$ I vs control group; $* * p<0.0$ I vs TS group.

score and nodding numbers can objectively reflect the severity of tics. The autonomic activity test can reflect the exploration characteristics of each rat and can be used to quantitatively evaluate the excitability, exploration behavior, and spontaneous behavior of rats. ${ }^{31}$ Here, we found that injection with IDPN remarkedly increased the stereotypy score, nodding numbers, number of times to enter the central area, and total movement distance of the rats compared with those of the control group and produced persistent behavioral syndrome imitating TS. Tiapride (Tia) is a selective dopamine receptor (D2R) antagonist that has been proved to antagonize the symptoms of dyskinesia caused by injection of dopamine in the striatum. ${ }^{30}$ Tia has been used to treat TS for many years and possesses anti-tic effects, as reported in clinical and experimental studies. ${ }^{32,33}$ Therefore, Tia was used as a positive control drug for a comparison with the effect of Gas on TS. Here, we found that Gas and Tia showed a significant improvement in stereotypy score, nodding numbers, number of times to enter the central area, and the total movement distance of the rats with TS, which indicated that both Gas and Tia could ameliorate the severity of behavioral symptoms in the rats with TS. Our findings are consistent with the results of previous studies showing that Gas and Tia have similar therapeutic effects on the behaviors of rats with $\mathrm{TS}^{24,25}$

Currently, an increasing number of studies have demonstrated that malfunction of CSTS circuits, which are involved in cognitive, sensorimotor, and emotional functions, may be related to the development of a variety of neuropsychiatric diseases, including TS. ${ }^{34,35}$ Abnormal amino acid neurotransmitters, such as Glu and GABA, involved in the CSTS circuits, are closely associated with the occurrence of TS. ${ }^{13,14}$ GABA is the primary inhibitory neurotransmitter in the CNS of mammals. Puts et al found reduced GABA concentrations in patients with TS and, importantly, that there was a negative correlation between GABA concentration and 
A

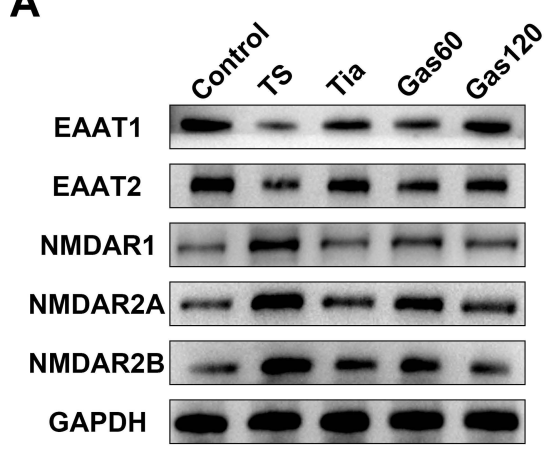

D

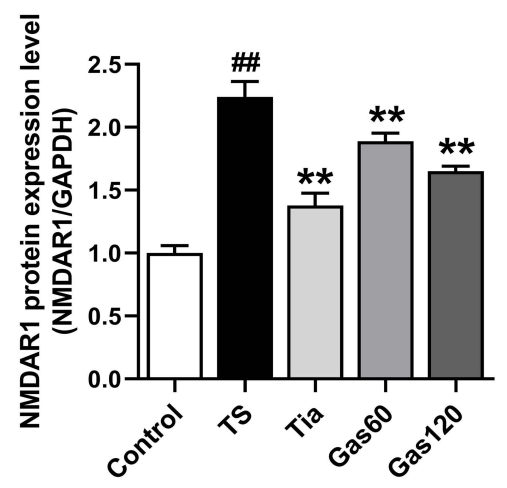

B

E
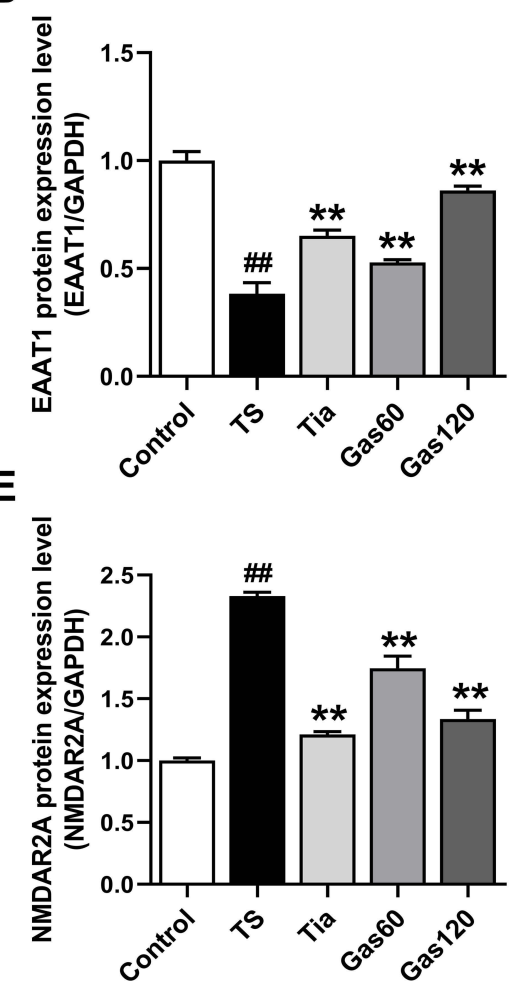

C

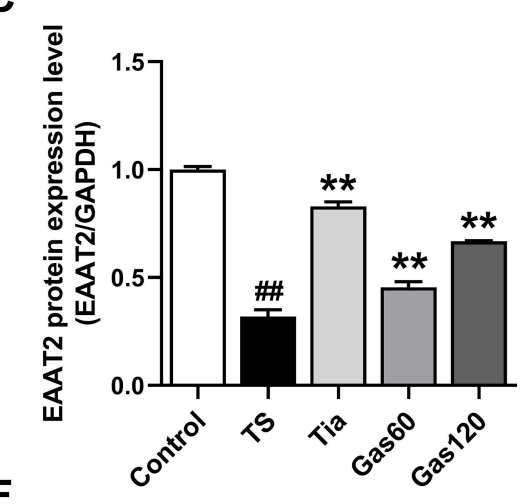

$\mathbf{F}$

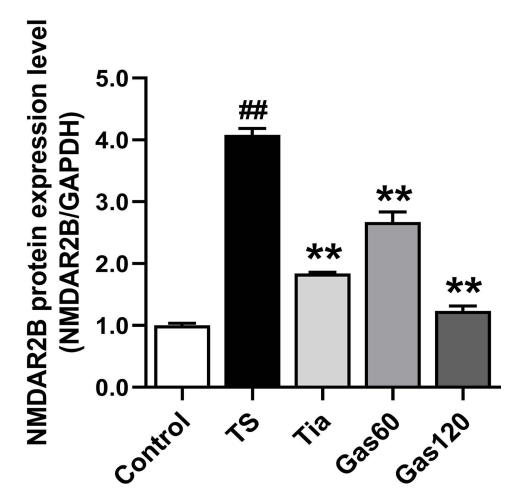

Figure 6 Effects of Gas treatment on the protein expression of EAATI, EAAT2, NMDARI, NMDAR2A and NMDAR2B in the striatum of the rats with TS induced by IDPN. Notes: (A) Representative protein bands of EAATI, EAAT2, NMDARI, NMDAR2A and NMDAR2B of each group of rats in the striatum. (B) The protein expression of EAATI in the striatum detected by Western blot. (C) The protein expression of EAAT2 in the striatum detected by Western blot. (D) The protein expression of NMDARI in the striatum detected by Western blot. (E) The protein expression of NMDAR2A in the striatum detected by Western blot. (F) The protein expression of NMDAR2B in the striatum detected by Western blot. The levels of these proteins were measured as described in materials and methods. Each bar represented the mean \pm SD. ${ }^{\#} p<0.01$ vs control group; $* *<<0.01$ vs TS group.

tic severity. ${ }^{36}$ However, our study demonstrated that administration of IDPN caused significantly increased GABA concentrations in the striatum of the rats compared with those of the controls. Although our results were contradictory to the findings of Puts et al, they were consistent with the findings of Chen et al, in which increased GABA concentrations was observed in IDPN-treated rats. ${ }^{30}$ The increased GABA concentration in the striatum, as observed in the present and previous studies, was possibly due to an adaptive response to inhibit the hyperactivity that caused repetitive and involuntary motor in rats with TS. In addition, our results demonstrated that the increased GABA levels could not be decreased by Gas treatment. Therefore, GABA might not participate in the mechanism of drug action of Gas. Glu is the primary excitatory neurotransmitter in the mammalian brain, and it is associated with essential brain functions. ${ }^{37}$ Numerous neurological disorders, such as neurodegenerative diseases, epilepsy, cerebrovascular diseases, and TS, are closely related to abnormal concentrations of Glu. ${ }^{37}$ It is widely accepted that Glu shows an increasing trend in patients with TS, resulting in an overexcited state in the CNS. As reported in previous studies, the levels of Glu in patients were significantly increased, as evaluated by magnetic resonance measurements. ${ }^{16}$ When the excess release of Glu is inhibited, it can effectively ameliorate the development of TS and its complications. Animal studies showed that there was an increase in the levels of Glu in the striatum of TS model rats. ${ }^{38}$ Interestingly, consistent with previous studies, our study demonstrated that the Glu levels in the striatum showed a significant increase after injection with IDPN compared with that of the control group. Furthermore, our results showed that treatment with Gas and Tia could efficiently decrease the Glu levels in the striatum. Moreover, the alleviation of the severity of behavioral symptoms in the rats with TS treated with Gas was paralleled by a prominent decrease in Glu levels in the striatum. This study confirmed that a high level of Glu was closely related to the pathogenesis of TS. 

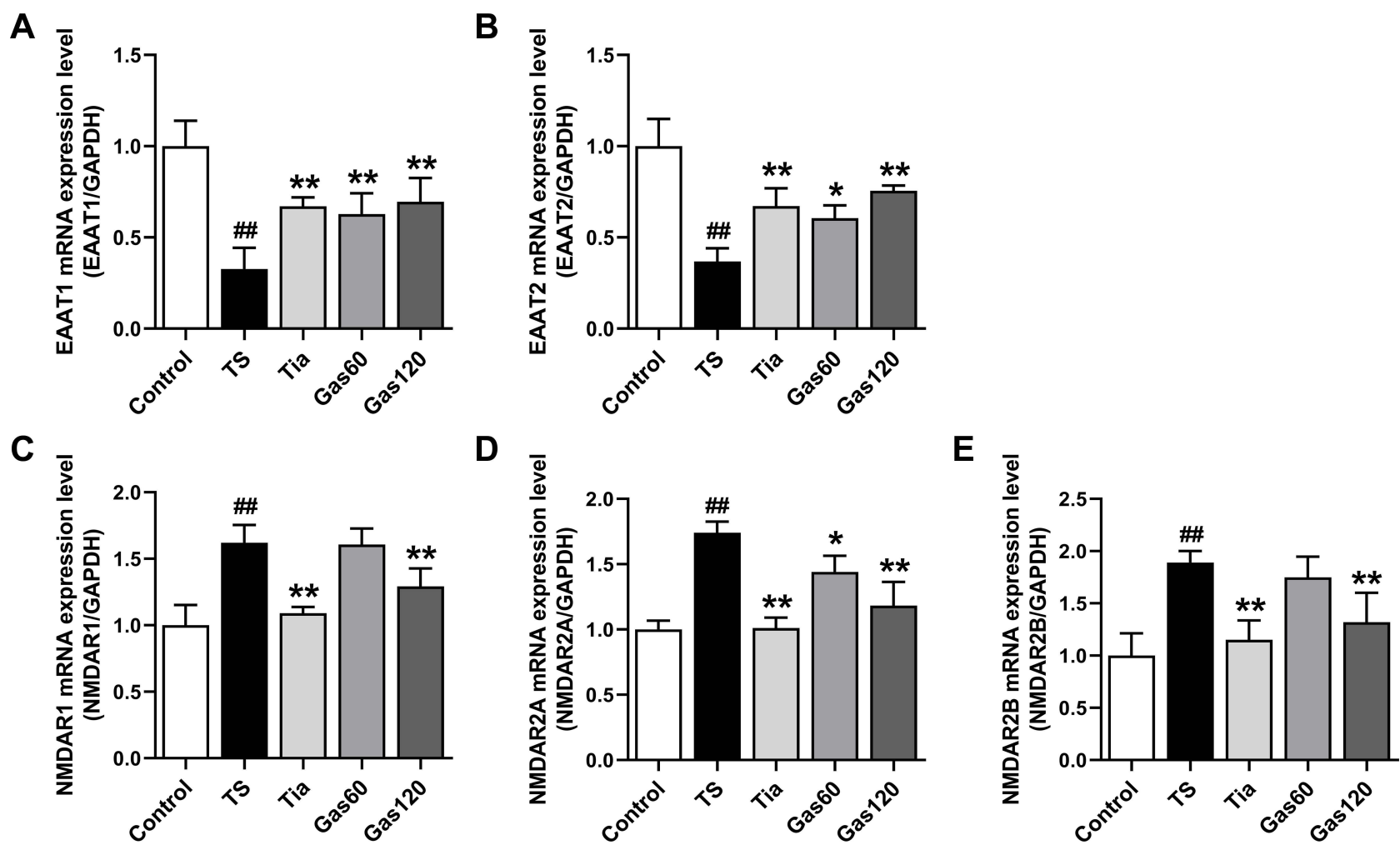

Figure 7 Effects of Gas treatment on the mRNA expression of EAATI, EAAT2, NMDARI, NMDAR2A and NMDAR2B in the striatum of the rats with TS induced by IDPN. Notes: (A) The mRNA expression of EAATI in the striatum detected by qRT-PCR. (B) The mRNA expression of EAAT2 in the striatum detected by qRT-PCR. (C) The mRNA expression of NMDARI in the striatum detected by qRT-PCR. (D) The mRNA expression of NMDAR2A in the striatum detected by qRT-PCR. (E) The mRNA expression of NMDAR2B in the striatum detected by qRT-PCR. The expressions of these mRNAs were measured as described in materials and methods. Each bar represented the mean $\pm S D$. ${ }^{\#} p<0.01$ vs control group; ${ }^{*} p<0.05, * * p<0.01$ vs TS group.

The excessive accumulation of extracellular Glu indicated that the clearance of Glu might be disrupted in rats with TS induced by IDPN. EAATs, which can mediate the clearance of Glu, include a class of five transporters with different expression in neurons and glia cells. ${ }^{17}$ When Glu is released into the synaptic cleft, it is rapidly transferred out of the extracellular space by EAATs to prevent the excessive accumulation of Glu. It is a crucial functional element of glutamatergic synapses that EAATs take up Glu to maintain a suitable concentration of Glu in the synaptic cleft. Up to $80 \%$ of extracellular Glu is transferred out of the extracellular space through EAAT1 and EAAT2, which play a key role in the uptake of Glu, termination of excitability signals and neuronal damage protection. ${ }^{17}$ The dysfunction of EAAT1 and EAAT2 may lead to excessive accumulation of extracellular Glu and further activation of Glu receptors and excitatory transmission, which causes excitotoxicity of neurons and the occurrence of various neuropsychiatric disorders, including TS. Previous studies found increased Glu concentration and decreased expression of EAAT1 in the striatum of rats with TS induced by IDPN relative to those of the control group. ${ }^{38}$ Consistent with previous studies, our results showed that IDPN injection caused a significant increase in Glu concentration and a decrease in the protein and mRNA expression of EAAT1 and EAAT2 in the striatum compared with those of the control group. In the present study, the decreasing EAAT1 and EAAT2 expression suggested that the clearance of Glu was weakened in the rats with TS, leading to excessive accumulation of Glu. Thus, the inhibition of EAAT1 and EAAT2 might underlie the behavioral symptoms observed in the rats with TS induced by IDPN. In addition, we found an increase in EAAT1 and EAAT2 expression after treatment with Gas and Tia. Our results suggested that Gas could ameliorate tic-like symptoms by upregulating the expression of EAAT1 and EAAT2 to increase the clearance of Glu and maintain the concentration of extracellular Glu at a suitable level. These findings revealed that EAAT1 and EAAT2 might participate in the mechanism of Gas drug action.

Among Glu receptors, NMDARs have been proved to be associated with the occurrence of TS. NMDARs, which are widely distributed in the mammalian brain, constitute a main 
class of Glu receptors. ${ }^{39}$ NMDARs consist of NMDAR1, NMDAR2 and NMDAR3 subunits, and the physiology of NMDARs is the basis of brain development and function. ${ }^{39}$ NMDARs can mediate glutamatergic neurotransmission and play a key role in several basic functions in the CNS, such as the regulation of neurodevelopment and synaptic plasticity, excitotoxicity, cognitive processes, and learning and memory formation. ${ }^{18}$ NMDARs are closely related to the development of several neuropsychiatric disorders, including neurodegenerative disorders, autism, traumatic brain injury, and affective disorders. ${ }^{40,41}$ Neurons release Glu to the synaptic cleft, and extracellular Glu activates Glu receptors (including NMDARs) located in neurons and glial cells to regulate brain excitability. Nevertheless, under pathological conditions, excessive accumulation of extracellular Glu can over-activate NMDARs and lead to excitotoxicity, resulting in various neuropsychiatric disorders. As reported in a previous study, a significant increase in the stereotypy score and total movement distance in rats with TS induced by IDPN was paralleled by prominently increased Glu levels and over-activated NMDAR1 relative to those of the control group. ${ }^{42}$ In this study, we found that the expression of NMDARs including NMDAR1, NMDAR2A and
NMDAR2B were increased after IDPN injection, which might be the reason for the increases in the stereotypy score, nodding numbers, number of times to enter the central area and total movement distance. The Western blot and qRT-PCR findings confirmed that excessive accumulation of extracellular Glu could over-activate NMDAR1, NMDAR2A and NMDAR2B. In addition, our study showed that Gas treatment could depress the over-activated NMDAR1, NMDAR2A and NMDAR2B, suggesting that NMDAR1, NMDAR2A and NMDAR2B might participate in the mechanism of Gas drug action.

Gas is one of the major bioactive components isolated from the Rhizoma Gastrodiae. It has been reported that Gas has beneficial effects on many CNS diseases including TS. ${ }^{23-}$ ${ }^{25}$ Here, we found that Gas could ameliorate tic-like symptoms, including stereotypy score, nodding numbers, number of times to enter the central area and the total movement distance of the rats with TS induced by IDPN, which were consistent with previous research. ${ }^{24,25}$ In the present study, we further elaborated the anti-tics effects of Gas on the rats with TS induced by IDPN and the potential mechanism. Our results confirmed that Gas could decrease the Glu concentration in the

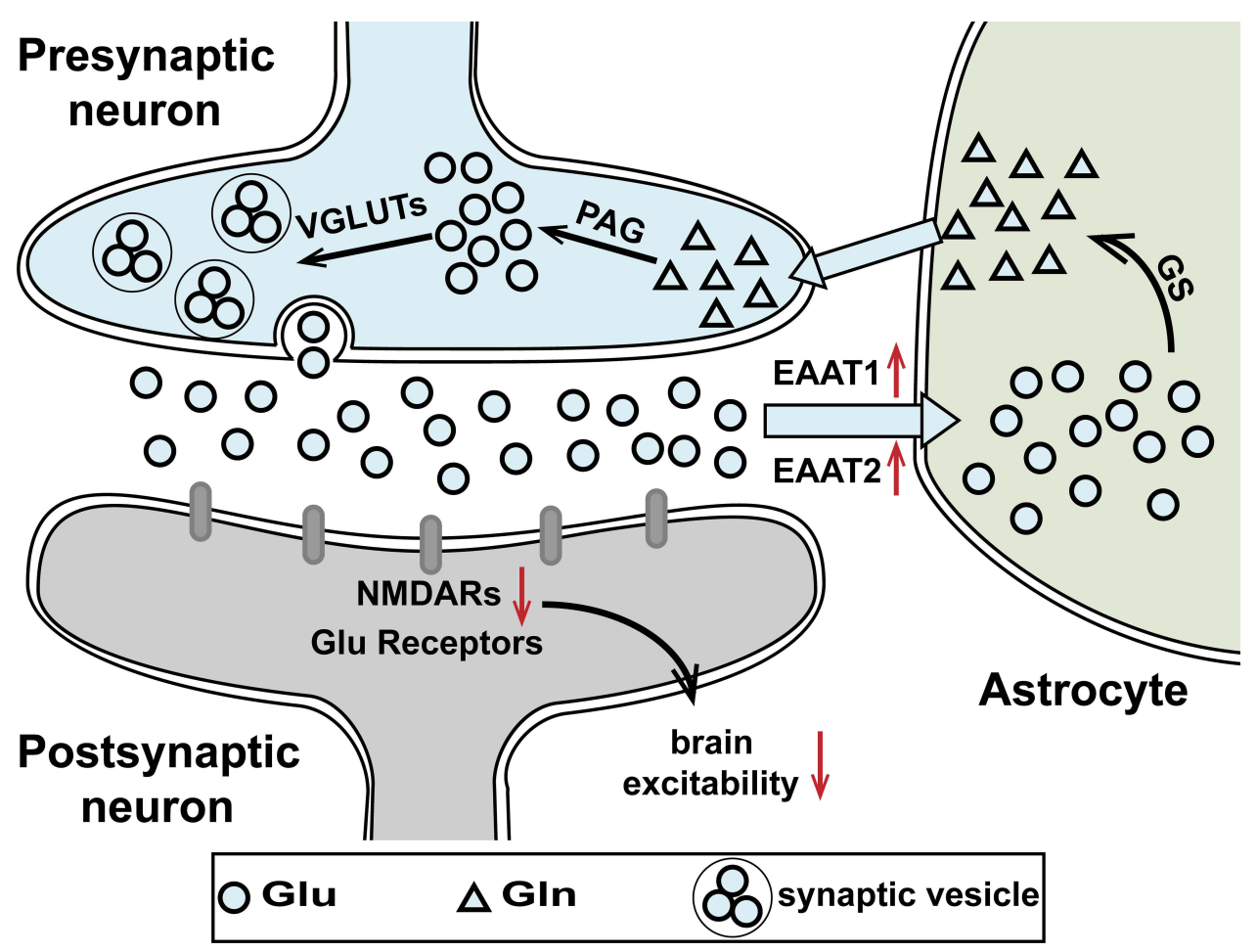

Figure 8 A schematic illustration of the proposed mechanism by which Gas alleviates Tourette syndrome in rats.

Notes: The red arrows indicate the possible mechanism of Gas. Gas might maintain the concentration of extracellular Glu at a suitable level by upregulating the expression of EAATI and EAAT2 to increase the clearance of Glu. The decreased Glu level might inhibit over-activation of NMDARI, NMDAR2A and NMDAR2B and depress glutamatergic neurotransmission and brain excitability.

Abbreviations: Glu, glutamate; Gln, glutamine; EAATI, Glutamate aspartate transporter (GLAST); EAAT2, Glial glutamate transporter (GLT-I); NMDARs, N-methyld-aspartate receptors; VGLUTs, vesicular glutamate transporters; GS, Glutamine Synthetase; PAG, phosphate activated glutaminase. 
striatum of the rats with TS induced by IDPN, which was consistent with the results using high performance liquid chromatography shown by previous research. ${ }^{24}$ We also found that the effect of Gas on decreasing extracellular Glu concentration was closely related to the downregulation of EAAT1 and EAAT2 expression. When the extracellular Glu concentration is suitable level, NMDARs might not be overactivated. Therefore, our findings showed a significant decrease in the protein and mRNA expression of NMDAR1, NMDAR2A and NMDAR2B after treatment with Gas. Together, these findings indicated that TS rats induced by IDPN could lead to tic-like symptoms, an increase in Glu concentration in the striatum, a decrease in EAAT1 and EAAT2 expression and an increase in NMDAR1, NMDAR2A and NMDAR2B expression, which could be altered by Gas treatment. EAAT1, EAAT2, NMDAR1, NMDAR2A and NMDAR2B might participate in the mechanism of Gas drug action. Our findings provide a pharmacological basis for Gas as a clinical treatment for patients with TS. A schematic illustration of the proposed mechanism for Gas to alleviate TS rats is shown in Figure 8.

However, this study also has some limitations. First, there are differences between rats and humans, so further trials conducted on patients with TS can provide more clinical information for TS treatment. Second, in addition to glutamatergic neurotransmission, other pathways may have a crucial effect on the development of TS. These questions need to be explored in further research.

\section{Conclusion}

Gas treatment might be effective in improving the behavioral dysfunction of rats with TS induced by IDPN. This beneficial effect of Gas was closely related to the decreased Glu concentration, upregulated expression of EAAT1 and EAAT2, and downregulated expression of NMDAR1, NMDAR2A and NMDAR2B in the striatum of rats with TS after Gas treatment, which could depress glutamatergic neurotransmission and brain excitability. These effects might be the reason that Gas treatment could improve the severity of behavioral symptoms of rats with TS induced by IDPN. Taken together, our findings provide a pharmacological basis for the advantageous effects of Gas treatment for patients with TS.

\section{Acknowledgments}

This study was supported by grants from the National Natural Science Foundation of China (No 81874486) and Shanghai Science and Technology Commission Research Project (No 18401902700; No 17401931200).

\section{Disclosure}

The authors report no conflicts of interest in this work.

\section{References}

1. Müller-Vahl KR, Bindila L, Lutz B, et al. Cerebrospinal fluid endocannabinoid levels in Gilles de la Tourette syndrome. Neuropsychopharmacology. 2020;45(8):1323-1329. doi:10.1038/ s41386-020-0671-6

2. Groth C, Mol Debes N, Rask CU, Lange T, Skov L. Course of Tourette syndrome and comorbidities in a large prospective clinical study. J Am Acad Child Adolesc Psychiatry. 2017;56(4):304-312. doi:10.1016/j.jaac.2017.01.010

3. Robertson MM. The prevalence and epidemiology of Gilles de la Tourette syndrome. Part 1: the epidemiological and prevalence studies. J Psychosom Res. 2008;65(5):461-472. doi:10.1016/j. jpsychores.2008.03.006

4. Scharf JM, Miller LL, Gauvin CA, Alabiso J, Mathews CA, BenShlomo Y. Population prevalence of Tourette syndrome: a systematic review and meta-analysis. Mov Disord. 2015;30(2):221-228. doi:10.1002/mds.26089

5. Hirschtritt ME, Lee PC, Pauls DL, et al. Lifetime prevalence, age of risk, and genetic relationships of comorbid psychiatric disorders in Tourette syndrome. JAMA Psychiatry. 2015;72(4):325-333. doi:10.1001/jamapsychiatry.2014.2650

6. Conte G, Valente F, Fioriello F, Cardona F. Rage attacks in Tourette syndrome and chronic tic disorder: a systematic review. Neurosci Biobehav Rev. 2020;119:21-36. doi:10.1016/j. neubiorev.2020.09.019

7. Robertson MM. A personal 35 year perspective on Gilles de la Tourette syndrome: prevalence, phenomenology, comorbidities, and coexistent psychopathologies. Lancet Psychiatry. 2015;2(1):68-87. doi:10.1016/S2215-0366(14)00132-1

8. Huisman-van Dijk HM, Schoot R, Rijkeboer MM, Mathews CA, Cath DC. The relationship between tics, OC, ADHD and autism symptoms: a cross- disorder symptom analysis in Gilles de la Tourette syndrome patients and family-members. Psychiatry Res. 2016;237:138-146. doi:10.1016/j.psychres.2016.01.051

9. Verté S, Geurts HM, Roeyers H, Oosterlaan J, Sergeant JA. Executive functioning in children with autism and Tourette syndrome. Dev Psychopathol. 2005;17(2):415-445. doi:10.1017/ S0954579405050200

10. Channon S, Gunning A, Frankl J, Robertson MM. Tourette's syndrome (TS): cognitive performance in adults with uncomplicated TS. Neuropsychology. 2006;20(1):58-65. doi:10.1037/0894-4105.20.1.58

11. Liu S, Zheng L, Zheng X, Zhang X, Yi M, Ma X. The subjective quality of life in young people with Tourette syndrome in China. J Atten Disord. 2017;21(5):426-432. doi:10.1177/1087054713518822

12. Martino D, Dale RC, Gilbert DL, Giovannoni G, Leckman JF. Immunopathogenic mechanisms in tourette syndrome: a critical review. Mov Disord. 2009;24(9):1267-1279. doi:10.1002/mds.22504

13. Singer HS, Morris C, Grados M. Glutamatergic modulatory therapy for Tourette syndrome. Med Hypotheses. 2010;74(5):862-867. doi:10.1016/j.mehy.2009.11.028

14. Ramamoorthi K, Lin Y. The contribution of GABAergic dysfunction to neurodevelopmental disorders. Trends Mol Med. 2011;17 (8):452-462. doi:10.1016/j.molmed.2011.03.003

15. Reiner A, Levitz J. Glutamatergic signaling in the central nervous system: ionotropic and metabotropic receptors in concert. Neuron. 2018;98(6):1080-1098. doi:10.1016/j.neuron.2018.05.018

16. Mahone EM, Puts NA, Edden RAE, Ryan M, Singer HS. GABA and glutamate in children with Tourette syndrome: a (1)H MR spectroscopy study at 7T. Psychiatry Res Neuroimaging. 2018;273:46-53. doi:10.1016/j.pscychresns.2017.12.005 
17. Malik AR, Willnow TE. Excitatory amino acid transporters in physiology and disorders of the central nervous system. Int J Mol Sci. 2019;20(22):5671. doi:10.3390/ijms20225671

18. Traynelis SF, Wollmuth LP, McBain CJ, et al. Glutamate receptor ion channels: structure, regulation, and function. Pharmacol Rev. 2010;62(3):405-496.

19. Vyklicky V, Korinek M, Smejkalova T, et al. Structure, function, and pharmacology of NMDA receptor channels. Physiological Research. 2014;63(Suppl 1):S191-203. doi:10.33549/physiolres.932678

20. Skowrońska K, Obara-Michlewska M, Zielińska M, Albrecht J. NMDA receptors in astrocytes: in search for roles in neurotransmission and astrocytic homeostasis. Int J Mol Sci. 2019;20(2):309. doi:10.3390/ijms20020309

21. Goldsmith PJ. NMDAR PAMs: multiple chemotypes for multiple binding sites. Curr Top Med Chem. 2019;19(24):2239-2253. doi:10.2174/1568026619666191011095341

22. Che F, Zhang Y, Wang G, Heng X, Liu S, Du Y. The role of GRIN2B in Tourette syndrome: results from a transmission disequilibrium study. J Affect Disord. 2015;187:62-65. doi:10.1016/j.jad.2015.07.036

23. Liu Y, Gao J, Peng M, et al. A review on central nervous system effects of gastrodin. Front Pharmacol. 2018;9:24. doi:10.3389/ fphar.2018.00024

24. Long H, Ruan J, Zhang M, Wang C, Huang Y. Gastrodin alleviates Tourette syndrome via Nrf-2/HO-1/HMGB1/NF-кB pathway. J Biochem Mol Toxicol. 2019;33(10):e22389. doi:10.1002/jbt.22389

25. Long H, Wang C, Ruan J, Zhang M, Huang Y. Gastrodin attenuates neuroinflammation in DOI-induce Tourette syndrome in rats. J Biochem Mol Toxicol. 2019;33(5):e22302. doi:10.1002/jbt.22302

26. Peng Z, Wang $H$, Zhang $R$, et al. Gastrodin ameliorates anxiety-like behaviors and inhibits IL-1 $\beta$ level and p38 MAPK phosphorylation of hippocampus in the rat model of posttraumatic stress disorder. Physiological Research. 2013;62(5):537-545. doi:10.33549/ physiolres.932507

27. Chen PJ, Sheen LY. Gastrodiae Rhizoma (天麻 tiān má): a review of biological activity and antidepressant mechanisms. J Tradit Complement Med. 2011;1(1):31-40. doi:10.1016/S2225-4110(16)30054-2

28. Peng Z, Wang S, Chen G, et al. Gastrodin alleviates cerebral ischemic damage in mice by improving anti-oxidant and anti-inflammation activities and inhibiting apoptosis pathway. Neurochem Res. 2015;40(4):661-673. doi:10.1007/s11064-015-1513-5

29. Chen L, Liu X, Wang H, Qu M. Gastrodin attenuates pentylenetetrazole-induced seizures by modulating the mitogen-activated protein kinase-associated inflammatory responses in mice. Neurosci Bull. 2017;33(3):264-272. doi:10.1007/s12264-016-0084-Z

30. Chen J, Leong PK, Leung HY, et al. A Chinese herbal formulation, Xiao-Er-An-Shen decoction, attenuates tourette syndrome, possibly by reversing abnormal changes in neurotransmitter levels and enhancing antioxidant status in mouse brain. Front Pharmacol. 2019;10:812. doi:10.3389/fphar.2019.00812
31. Liu Y, Ding XF, Wang XX, et al. Xiaoyaosan exerts antidepressant-like effects by regulating the functions of astrocytes and EAATs in the prefrontal cortex of mice. BMC Complement Altern Med. 2019;19(1):215. doi:10.1186/s12906-019-2613-6

32. Fekete S, Egberts K, Preissler T, et al. Estimation of a preliminary therapeutic reference range for children and adolescents with tic disorders treated with tiapride. Eur J Clin Pharmacol. 2021;77 (2):163-170. doi:10.1007/s00228-020-03000-0

33. Pringsheim T, Holler-Managan Y, Okun MS, et al. Comprehensive systematic review summary: treatment of tics in people with Tourette syndrome and chronic tic disorders. Neurology. 2019;92 (19):907-915. doi:10.1212/WNL.0000000000007467

34. Tremblay L, Worbe Y, Thobois S, Sgambato-Faure V, Féger J. Selective dysfunction of basal ganglia subterritories: from movement to behavioral disorders. Mov Disord. 2015;30(9):1155-1170. doi:10.1002/mds.26199

35. Lerner A, Bagic A, Simmons JM, et al. Widespread abnormality of the $\gamma$-aminobutyric acid-ergic system in Tourette syndrome. Brain. 2012;135(Pt 6):1926-1936. doi:10.1093/brain/aws104

36. Puts NA, Harris AD, Crocetti D, et al. Reduced GABAergic inhibition and abnormal sensory symptoms in children with Tourette syndrome. J Neurophysiol. 2015;114(2):808-817. doi:10.1152/jn.00060.2015

37. Marmiroli P, Cavaletti G. The glutamatergic neurotransmission in the central nervous system. Curr Med Chem. 2012;19(9):1269-1276. doi:10.2174/092986712799462711

38. Yu W, Shi X, Cui X, et al. Jian-Pi-Zhi-Dong-decoction regulates the expression of glutamate transporters to attenuate glutamate excitotoxicity and exerts anti-tics effects in Tourette syndrome model rats. Neuropsychiatr Dis Treat. 2018;14:3381-3392. doi:10.2147/NDT. S185169

39. Paoletti P, Bellone C, Zhou Q. NMDA receptor subunit diversity: impact on receptor properties, synaptic plasticity and disease. Nat Rev Neurosci. 2013;14(6):383-400. doi:10.1038/nrn3504

40. Lau CG, Zukin RS. NMDA receptor trafficking in synaptic plasticity and neuropsychiatric disorders. Nat Rev Neurosci. 2007;8 (6):413-426. doi:10.1038/nrn2153

41. Mony L, Kew JN, Gunthorpe MJ, Paoletti P. Allosteric modulators of NR2B-containing NMDA receptors: molecular mechanisms and therapeutic potential. $\mathrm{Br} \quad J$ Pharmacol. 2009;157(8):1301-1317. doi:10.1111/j.1476-5381.2009.00304.x

42. Zhang W, Wei L, Yu W, et al. Effect of Jian-Pi-Zhi-Dong decoction on striatal glutamate and $\gamma$-aminobutyric acid levels detected using microdialysis in a rat model of Tourette syndrome. Neuropsychiatr Dis Treat. 2016;12:1233-1242. doi:10.2147/NDT.S106330
Neuropsychiatric Disease and Treatment

\section{Publish your work in this journal}

Neuropsychiatric Disease and Treatment is an international, peerreviewed journal of clinical therapeutics and pharmacology focusing on concise rapid reporting of clinical or pre-clinical studies on a range of neuropsychiatric and neurological disorders. This journal is indexed on PubMed Central, the 'PsycINFO' database and CAS, an is the official journal of The International Neuropsychiatric Association (INA). The manuscript management system is completely online and includes a very quick and fair peer-review system, which is all easy to use. Visit http://www.dovepress.com/testimonials.php to read real quotes from published authors. 